\title{
Method of constructing exactly solvable chaosf
}

\author{
Ken Umeno \\ Frontier Research Program, The Institute of Physical and Chemical Research (RIKEN) \\ 2-1 Hirosawa, Wako, Saitama 351-01, Japan
}

\begin{abstract}
We present a new systematic method of constructing rational mappings as ergordic transformations with nonuniform invariant measures on the unit interval $\boldsymbol{I}=[0,1]$. As a result, we obtain a two-parameter family of rational mappings that have a special property in that their invariant measures can be explicitly written in terms of algebraic functions of parameters and a dynamical variable. Furthermore, it is shown here that this family is the most generalized class of rational mappings possessing the property of exactly solvable chaos on $\boldsymbol{I}$, including the Ulam=Neumann map $y=4 x(1-x)$. Based on the present method, we can produce a series of rational mappings resembling the asymmetric shape of the experimentally obtained first return maps of the Beloussof-Zhabotinski chemical reaction, and we can match some rational functions with other experimentally obtained first return maps in a systematic manner.

05.45+b, 04.20.Jb
\end{abstract}

*To appear in Phys. Rev.E(1997) 
Characterizing invariant measures for explicit nonlinear dynamical systems is a fundamental problem which connects dynamical theory with statistics and statistical mechanics. In some cases, it would be desirable to to characterize ergordic invariant measures for simple chaotic dynamical systems. However, in the cases of chaotic dynamical systems, such attempts to obtain explicit invariant measures have rarely been made. One well-known exception is the logistic map $Y=4 X(1-X) \equiv f_{0}(X)$ on $\boldsymbol{I}=[0,1]$ given by Ulam and von Neumann in the late 1940's [1].

The Ulam=Neumann dynamical system $x_{i}=f_{0}\left(x_{i-1}\right)$ has an ergordic measure $\mu(d x)=$ $\frac{d x}{\pi \sqrt{x(1-x)}}$ such that the time averages of a function $Q(x)$ can be explicitly computed by the formula $\lim _{N \rightarrow \infty} \sum_{i=0}^{N-1} \frac{1}{N} Q\left(x_{i}\right)=\int_{0}^{1} \frac{Q(x) d x}{\pi \sqrt{x(1-x)}}$ for almost all initial conditions $x_{0} \in \boldsymbol{I}$. The first attempt to generalize the Ulam=Neumann map within a set of rational functions was made by Katsura and Fukuda in 1985 [2]. The Katsura and Fukuda model is written as

$$
Y=\frac{4 X(1-X)(1-l X)}{\left(1-l X^{2}\right)^{2}} \equiv f_{l}(X)
$$

for $0 \leq l<1$. Clearly, the Ulam=Neumann map can be regarded as a special case of Katsura $=$ Fukuda systems where the parameter $l$ is set to 0 . The author showed [3] that the Katsura=Fukuda mappings (1) also have ergordic measures which can be written explicitly as

$$
\mu(d x)=\rho(x) d x=\frac{d x}{2 K(l) \sqrt{x(1-x)(1-l x)}},
$$

where $K(l)$ is the elliptic integral of the first kind $(g=1)$ given by $K(l)=\int_{0}^{1} \frac{d u}{\sqrt{\left(1-u^{2}\right)\left(1-l u^{2}\right)}}$. It is known [2] that the Katsura=Fukuda systems and the Ulam=Neumann system also have explicit solutions in terms of the Jacobi sn elliptic function, as

$$
x_{n}=\operatorname{sn}^{2}\left(K(l) 2^{n} \theta_{0}\right), \quad \theta_{0} \in \boldsymbol{I},
$$

where $\sqrt{l}$ corresponds to the modulus of Jacobi elliptic functions. The validity of the formulae of the general solutions (3) is easily checked using the duplication formula [4] of the Jacobi sn elliptic function 


$$
s n(2 u)=\frac{2 s n(u) \sqrt{\left(1-s n^{2}(u)\right)\left(1-l s n^{2}(u)\right)}}{\left(1-l s n^{4}(u)\right)} .
$$

Because the Ulam=Neumann system and Katsura=Fukuda systems have not only exact solutions (3) but also explicitly written ergordic invariant measures (2), we call a dynamical system exactly solvable chaos if it has both of these properties.

Thus, it is of great interest to investigate whether we can generalize the Ulam=Neumann system and the Katsura=Fukuda systems further within a set of rational functions, maintaining the property of exactly solvable chaos. The main purpose of the present letter is to show that by using the addition formulas of elliptic functions we can construct a twoparameter family of rational mappings of exactly solvable chaos, and at the same time, that there is a certain limitation to generalizing this family within a set of rational functions.

Our results reported here [5] concern the following rational transformations

$$
Y=f_{l, m}(X)=\frac{4 X(1-X)(1-l X)(1-m X)}{1+A X^{2}+B X^{3}+C X^{4}} \in I,
$$

where $A=-2(l+m+l m), B=8 l m, C=l^{2}+m^{2}-2 l m-2 l^{2} m-2 l m^{2}+l^{2} m^{2}$, and $X \in I$. The parameters $l$ and $m$ are arbitrary real numbers satisfying the condition $-\infty<m \leq l<1$. Figure 1(a) shows various shapes of the proposed mappings (5). Surprisingly, some rational maps in (5) strongly resemble the asymmetric shape of the experimentally obtained first return maps of the Beloussof-Zhabotinski chemical reaction. Here, we will prove the following statement. The two-parameter family of rational mappings (5) is also exactly solvable chaos such that the dynamical systems $x_{i+1}=f_{l, m}\left(x_{i}\right)$ (5) have ergordic invariant measures explicitly given by

$$
\mu(d x)=\rho(x) d x=\frac{d x}{2 K(l, m) \sqrt{x(1-x)(1-l x)(1-m x)}},
$$

where $K$ is given by the integrals

$$
K(l, m)=\int_{0}^{1} \frac{d u}{\sqrt{\left(1-u^{2}\right)\left(1-l u^{2}\right)\left(1-m u^{2}\right)}},
$$

and it has general solutions explicitly given by 


$$
x_{n}=s^{2}\left(K(l, m) 2^{n} \theta_{0}\right) \quad \theta_{0} \in \boldsymbol{I} .
$$

We prove this by explicitly computing the duplication formula of the following degenerated hyperelliptic function $s(x)$ defined by

$$
s^{-1}(x)=\int_{0}^{x} \frac{d u}{\sqrt{\left(1-u^{2}\right)\left(1-l u^{2}\right)\left(1-m u^{2}\right)}} .
$$

Here, degenerated means that this hyperelliptic integral of the R.H.S. of Eq. (9) can be reduced to a certain elliptic integral by a rational change of variables. Although the reduction of Abelian integrals of genus $g \geq 2$ to elliptic functions was intensively studied in the 19th century by Jacobi, Weierstrass, Königsberger, Kovalevskaya and others, it was only in the 1980's that the theory of the reduction was successfully applied to physics for obtaining, for example, explicit periodic solutions in terms of elliptic functions for the Korteweg-de Vries $(\mathrm{KdV})$ equation and the sin-Gordon equation [6]. Let us consider reduction of the hyperelliptic integrals (9) as

$$
s^{-1}(x)=\int_{0}^{x} \frac{d u}{\sqrt{\left(1-u^{2}\right)\left(1-l u^{2}\right)\left(1-m u^{2}\right)}}=\int_{0}^{x^{2}} \frac{d v}{2 \sqrt{v(1-v)(1-l v)(1-m v)}}
$$

where $u^{2}=v$. Thus, we can write the degenerated hyperelliptic function $s(x)$ in terms of the Weierstrass elliptic functions. The Weierstrass elliptic function $\wp(u)$ of $u \in \boldsymbol{C}$ is defined by

$$
\wp(u)=\frac{1}{u^{2}}+\sum_{j, k}^{\prime}\left\{\frac{1}{\left(u-2 j \omega_{1}-2 k \omega_{2}\right)^{2}}-\frac{1}{\left(2 j \omega_{1}+2 k \omega_{2}\right)^{2}}\right\}
$$

where the symbol $\sum^{\prime}$ means that the summation is made over all combinations of integers $j$ and $k$, except for the combination $j=k=0$, and $2 \omega_{1}$ and $2 \omega_{2}$ are periods of the function $\wp(u)$ [4]. The Weierstrass elliptic function $\wp(u)$ satisfies the differential equation

$$
\left(\frac{d \wp(x)}{d x}\right)^{2}=4 \wp^{3}(x)-g_{2} \wp(x)-g_{3}
$$

with the invariants $g_{2}\left(\omega_{1}, \omega_{2}\right)=60 \sum_{j, k}^{\prime} \frac{1}{\left(j \omega_{1}+k \omega_{2}\right)^{4}}$ and $g_{3}\left(\omega_{1}, \omega_{2}\right)=140 \sum_{j, k}^{\prime} \frac{1}{\left(j \omega_{1}+k \omega_{2}\right)^{6}}$ [4]. Let $e_{1}, e_{2}$ and $e_{3}$ be the roots of the equation $4 z^{3}-g_{2} z-g_{3}=0$; that is, 


$$
e_{1}+e_{2}+e_{3}=0, \quad e_{1} e_{2}+e_{2} e_{3}+e_{3} e_{1}=-\frac{g_{2}}{4}, \quad e_{1} e_{2} e_{3}=\frac{g_{3}}{4}
$$

The discriminant $\Delta$ of the function $\wp(u)$ is given by $\Delta=g_{2}^{3}-27 g_{3}^{2}$. If $\Delta>0$, all roots $e_{1}, e_{2}$ and $e_{3}$ of the equation $4 z^{3}-g_{2} z-g_{3}=0$ are real. Thus, we can assume that $e_{1}>e_{2}>e_{3}$. In the case that $\Delta>0$, it is known that the periods $\omega_{1}$ and $\omega_{2}$ are written simply as

$$
\omega_{1}=\int_{e_{1}}^{\infty} \frac{d z}{\sqrt{4 z^{3}-g_{2} z-g_{3}}}, \quad \omega_{2}=i \int_{-\infty}^{e_{3}} \frac{d z}{\sqrt{g_{3}+g_{2} z-4 z^{3}}}
$$

Using the transformation of the variable as $v=-\frac{(1-l)(1-m)}{y-\frac{2 l+2 m-3 l m-1}{3}}+1$, we can rewrite $s^{-1}(x)$ in Eq.(10) as

$$
\int_{0}^{x^{2}} \frac{d v}{2 \sqrt{v(1-v)(1-l v)(1-m v)}}=\int_{\frac{2-l-m}{3}}^{\frac{2 l+2 m-3 l m-1}{3}+\frac{(1-l)(1-m)}{1-x^{2}}} \frac{d y}{\sqrt{4 y^{3}-g_{2} y-g_{3}}},
$$

where $g_{2}=\frac{4\left(1-l+l^{2}-m+m^{2}-l m\right)}{3}$ and $g_{3}=\frac{4(2-l-m)(2 l-m-1)(2 m-l-1)}{27}$. We note here that $4 y^{3}-$ $g_{2} y-g_{3}$ can be factored as

$$
4 y^{3}-g_{2} y-g_{3}=4\left(y-\frac{2-l-m}{3}\right)\left(y-\frac{2 l-m-1}{3}\right)\left(y-\frac{2 m-l-1}{3}\right) .
$$

We set $e_{1}=\frac{2-l-m}{3}>e_{2}=\frac{2 l-m-1}{3}>e_{3}=\frac{2 m-l-1}{3}$. Thus, using the integral representation of the period $\omega_{1}(14)$ and the differential equation (12) for the Weierstrass elliptic function, $s(x)$ can be written explicitly in terms of the Weierstrass elliptic function as

$$
s^{2}(x)=1-\frac{(1-l)(1-m)}{\wp\left(\omega_{1}-x\right)-\frac{2 l+2 m-3 l m-1}{3}} .
$$

The function $s^{2}(x)$ also has the same periods $\omega_{1}$ and $\omega_{2}$ computed using formula (14). It is noted here that because

$$
\Delta \equiv g_{2}^{3}-27 g_{2}^{2}=16(1-l)^{2}(1-m)^{2}(l-m)^{2}>0
$$

for $-\infty<m<l<1$, the period $2 \omega_{1}$ is always real, while the period $2 \omega_{2}$ is always pure imaginary. Using the addition formula,

$$
\wp(z+y)=\frac{1}{4}\left\{\frac{\wp^{\prime}(z)-\wp^{\prime}(y)}{\wp(z)-\wp(y)}\right\}^{2}-\wp(z)-\wp(y)
$$

and the duplication formula, 


$$
\wp(2 z)=\frac{1}{4}\left\{\frac{\wp^{\prime \prime}(z)}{\wp^{\prime}(z)}\right\}^{2}-2 \wp(z),
$$

for the Weierstrass elliptic function $\wp(u)$ [ब], we finally obtain the explicit duplication formula of $s(x)$ as

$$
s^{2}(2 x)=\frac{4 s^{2}(x)\left(1-s^{2}(x)\right)\left(1-l s^{2}(x)\right)\left(1-m s^{2}(x)\right)}{1+A s^{4}(x)+B s^{6}(x)+C s^{8}(x)},
$$

where $A=-2(l+m+l m), B=8 l m$ and $C=l^{2}+m^{2}-2 l m-2 l^{2} m-2 l m^{2}+l^{2} m^{2}$. If we set $X=s^{2}(x)$ and $Y=s^{2}(2 x)$, we obtain system (5) as $Y=f_{l, m}(X)$.

Using the relations

$$
s^{2}\left(\omega_{1} \cdot 2 \theta\right)=f_{l, m}\left(s^{2}\left(\omega_{1} \theta\right)\right), \quad s^{2}\left(\omega_{1} \cdot(2-2 \theta)\right)=f_{l, m}\left(s^{2}\left(\omega_{1} \theta\right)\right),
$$

for $\theta \in[0,1]$ and by defining the homeomorphism of $[0,1]$ into itself given by $\phi_{l, m}(x)=$ $\frac{1}{\omega_{1}} s^{-1}(\sqrt{x})$, we derive the tent map $\tilde{f}(x)=\phi_{l, m} \circ f_{l, m} \circ \phi_{l, m}^{-1}$ as

$$
\tilde{f}(x)=2 x \quad \text { for } \quad x \in\left[0, \frac{1}{2}\right], \quad \tilde{f}(x)=2-2 x \quad \text { for } \quad x \in\left[\frac{1}{2}, 1\right] .
$$

Because this tent map (23) is clearly ergordic and preserves the Lebesgue measure, the map $f_{l, m}$ preserves the measures

$$
\mu(d x)=\frac{d \phi_{l, m}}{d x} d x=\frac{d x}{2 K(l, m) \sqrt{x(1-x)(1-l x)(1-m x)}} .
$$

This measure (24) is absolutely continuous with respect to the Lebsegue measure, which implies that the Kolmogorov-Sinai entropy $h(\mu)$ is equivalent to the Lyapunov exponent of $\log 2$ from the Pesin identity [0], and that the measure (24) is a physical one in the sense that it is the Sinai-Ruelle-Bowen (SRB) measure such that for almost all initial conditions $x_{0}$, the time averages $\lim _{N \rightarrow \infty} \frac{1}{N} \sum_{i=0}^{N-1} \delta\left(x-x_{i}\right)$ reproduce the invariant measure $\mu(d x)$ []].

In the same way, we can construct generalized cubic maps $f_{l, m}^{(3)}$ from the triplication formula $s^{2}(3 x)=f_{l, m}^{(3)}\left(s^{2}(x)\right)$ as

$$
Y=f_{l, m}^{(3)}(X)=\frac{X\left(-3+4 X+\sum_{i=1}^{4} A_{i} X^{i}\right)^{2}}{1+\sum_{i=2}^{9} B_{i} X^{i}},
$$


where $A_{1}, \cdots, A_{4}$ and $B_{2}, \cdots, B_{9}$ are polynomial functions in the parameters $l$ and $m$ which vanish for $l=m=0$ [B] . The generalized cubic map $f_{l, m}^{(3)}$ has the same invariant measures (24) because the relation $\tilde{f}^{(3)}(x)=\phi_{l, m} \circ f_{l, m}^{(3)} \circ \phi_{l, m}^{-1}$ holds for the piecewise-linear map $\tilde{f}^{(3)}(x)=3 x$ for $0 \leq x \leq \frac{1}{3},-3 x+2$ for $\frac{1}{3} \leq x \leq \frac{2}{3}$ and $3 x-2$ for $\frac{2}{3} \leq x \leq 1$. If we set $l=m=0$, this rational mapping is reduced to the cubic map $Y=X(3-4 X)^{2}$ as a special case of Chebyshev maps obtained by Adler and Rivlin [9]. Thus, we can obtain generalized Chebyshev maps as rational functions $f_{l, m}^{(p)}$ from the addition formulas $s^{2}(p x)=f_{l, m}^{(p)}\left(s^{2}(x)\right)$, which have the same invariant measures (24). The shapes of generalized cubic maps are depicted in Fig. 1(b). Based on the case of Beloussof-Zhabotinski map, we predict here that some rational mappings (25), such as that shown in Fig. 1(b), can resemble the first return maps experimentally constructed from some unknown chaotic phenomena.

Are there more generalized rational mappings that possess the properties of exactly solvable chaos such as (5)? The exact solvability of the present rational mappings (5) and (25) is due to the fact that $s^{2}(x)$ in Eq. (17) is a rational function of the Weierstrass elliptic function $\wp(u)$ having the addition formula and the real period $\omega_{1}$. For an arbitrary set of parameters $e_{1}, e_{2}\left(<e_{1}\right)$, and $e_{3}\left(=-e_{1}-e_{2}\right)$ which determine the Weierstrass elliptic function $\wp(u)$ with the real period, there exists a set of parameters $l$ and $m$ of (5) given by $l=1-\left(e_{1}-e_{2}\right)$ and $m=1-\left(2 e_{1}-2 e_{2}\right)<l$; i.e., the mapping $h:\left(e_{1}, e_{2}\right) \mapsto(l, m)$ is a bijection. In other words, the family $\left\{f_{l, m}\right\}$ has one-to-one correspondence to the set of Weierstrass elliptic functions $\{\wp(u)\}$ with real periods. Furthermore, any elliptic function $w(u)$ can be expressed in terms of Weierstrassian elliptic functions $\wp(u)$ and $\wp(u)^{\prime}$ with the same periods, the expression being rational in $\wp(u)$ and linear in $\wp^{\prime}(u)$ [4. 10. Since it is known [11] from the Weierstrassian theorem that the class of functions $h(u)$ having the algebraic addition formulae [12 including the duplication formulae is exactly the class of algebraic functions of elliptic functions $\wp(u)$, which of course includes the class of algebraic functions of sin functions, we can say that this two-parameter family of dynamical systems essentially forms a maximal class in representing exactly solvable chaos induced by onedimensional rational mappings. 
In conclusion, we present a new method of constructing ergordic transformations related to rational functions with explicit nonuniform invariant measures using the addition formulas of elliptic functions. As a result, we systematically generalize the Ulam=Neumann (logistic) map and Chebyshev maps into two-parameter families of rational mappings. We also showed that constructing the more generalized family of rational mappings possessing explicit ergordic invariant measures has a certain limitation due to the Weierstrassian argument concerning the addition formulas for general algebraic functions. As for the applications, all of the constructive results given here for ergordic invariant measures by rational mappings can be directly used as nonlinear random number generators for the Monte Carlo methods.

The author would like to acknowledge Dr. K. Iguchi and Dr. A. Bobenko for useful discussions. Also, Professor T. Kohda and Professor S. Amari provided helpful suggestions. This work was supported by the RIKEN Special Researcher's Program to promote basic sciences. 


\section{FIGURES}

FIG. 1(a): (mono). The two-parameter family of exactly solvable chaos mappings (5) for $(l, m)=(0.1,-10),(0.1,-20), \cdots(0.1,-100)$. The asymmetric shapes of this class of mappings are very similar to the first return maps of the Beloussof-Zhabotinski chemical reaction. 
FIG. 1(b): (mono). The two-parameter family of exactly solvable chaos mappings (25) for $(l, m)=(0.1,-10),(0.1,-20), \cdots(0.1,-100)$. 


\section{REFERENCES}

[1] S.M. Ulam and J. von Neumann, Bull. Am. Math. Soc. 53, 1120 (1947).

[2] S. Katsura and W. Fukuda, Physica (Amsterdam) 130 A, 597 (1985).

[3] K. Umeno, "Inferring invariant measures of dynamical systems", IEICE Technical Report NC96-13 (1996).

[4] E.T. Whittaker and G.N. Watson, A Course of Modern Analysis (Cambridge University Press, 1935).

[5] This work was first reported as an oral presentation; K. Umeno, "Generalized UlamNeumann difference equations and statistics", at the International Workshop of Symmetries and Integrability of Difference Equations II held in Canterbury, England (July, 1996).

[6] E.D. Belkolos, A.I. Bobenko, V.B. Matveev and V.Z. Enol'skii, Russ. Math. Surv. 41(1986)1 and references cited therein.

[7] J.P. Eckmann and D. Ruelle, Rev. Mod. Phys. 57, 617 (1985).

[8] Precise forms of $A_{1}, \cdots, A_{4}$ and $B_{1}, \cdots, B_{9}$ are given as 


$$
\begin{aligned}
& A_{1}=4(l+m), A_{2}=-6(l+m+l m), A_{3}=12 l m, \\
& A_{4}=l^{2}+m^{2}-2 l m-2 l^{2} m-2 l m^{2}+l^{2} m^{2}, \\
& B_{2}=-12(l+m+l m), \\
& B_{3}=8\left(l+m+l^{2}+m^{2}+l^{2} m+l m^{2}+15 l m\right), \\
& B_{4}=6\left(5 l^{2}+5 m^{2}-26 l m-26 l^{2} m-26 l m^{2}+5 l^{2} m^{2}\right), \\
& B_{5}=24\left(-2 l^{2}-2 m^{2}-2 l^{3}-2 m^{3}+4 l m+7 l^{2} m+7 l m^{2}\right) \\
& +24\left(4 l^{3} m+4 l m^{3}+7 l^{2} m^{2}-2 l^{3} m^{2}-2 l^{2} m^{3}\right), \\
& B_{6}=4\left(4 l^{2}+4 m^{2}+4 l^{4}+4 m^{4}+17 l^{3}+17 m^{3}-8 l m\right) \\
& +4\left(-17 l^{2} m-17 l m^{2}-17 l^{3} m-17 l m^{3}-8 l^{4} m-8 l m^{4}\right) \\
& +4\left(4 l^{2} m^{4}+4 l^{4} m^{2}-17 l^{3} m^{2}-17 l^{2} m^{3}+17 l^{3} m^{3}-54 l^{2} m^{2}\right), \\
& B_{7}=24\left(-l^{3}-m^{3}-l^{4}-m^{4}+l^{2} m+l m^{2}-l^{3} m-l m^{3}\right) \\
& +24\left(l^{4} m+l m^{4}+4 l^{2} m^{2}+4 l^{3} m^{2}+4 l^{2} m^{3}\right) \\
& +24\left(l^{4} m^{2}+l^{2} m^{4}-l^{3} m^{3}-l^{4} m^{3}-l^{3} m^{4}\right), \\
& B_{8}=3\left(3 l^{4}+3 m^{4}+4 l^{3} m+4 l m^{3}+4 l^{4} m+4 l m^{4}-14 l^{2} m^{2}\right) \\
& +3\left(-4 l^{3} m^{2}-4 l^{2} m^{3}-4 l^{3} m^{3}-14 l^{4} m^{2}-14 l^{2} m^{4}+4 l^{4} m^{3}+4 l^{3} m^{4}+3 l^{4} m^{4}\right), \\
& B_{9}=8\left(-l^{4} m-l m^{4}+l^{3} m^{2}+l^{2} m^{3}+l^{4} m^{2}+l^{2} m^{4}-2 l^{3} m^{3}+l^{4} m^{3}+l^{3} m^{4}-l^{4} m^{4}\right) .
\end{aligned}
$$

[9] R.L. Adler and T.J. Rivlin, Proc. Am. Math. Soc. 15, 794 (1964).

[10] Thus, any elliptic function $w(u)$ has an algebraic relation with the Weierstrass elliptic function $\wp(u)$ with the same periods as $G(w, \wp)=0, G$ being a rational function of two variables.

[11] A. Hurwitz and R. Courant, Vorlesungen über allgemeine Funkitionentheorie und elliptische Funktionen (Springer-Verlag, Berlin, 1964).

[12] We say that a function $w(u)$ has an algebraic addition formula if there exists a polynomial function $G$ of three variables such that the relation $G\left(w\left(u_{1}+u_{2}\right), w\left(u_{1}\right), w\left(u_{2}\right)\right)=0$ holds for an arbitrary set of the variables $u_{1}$ and $u_{2}$. 


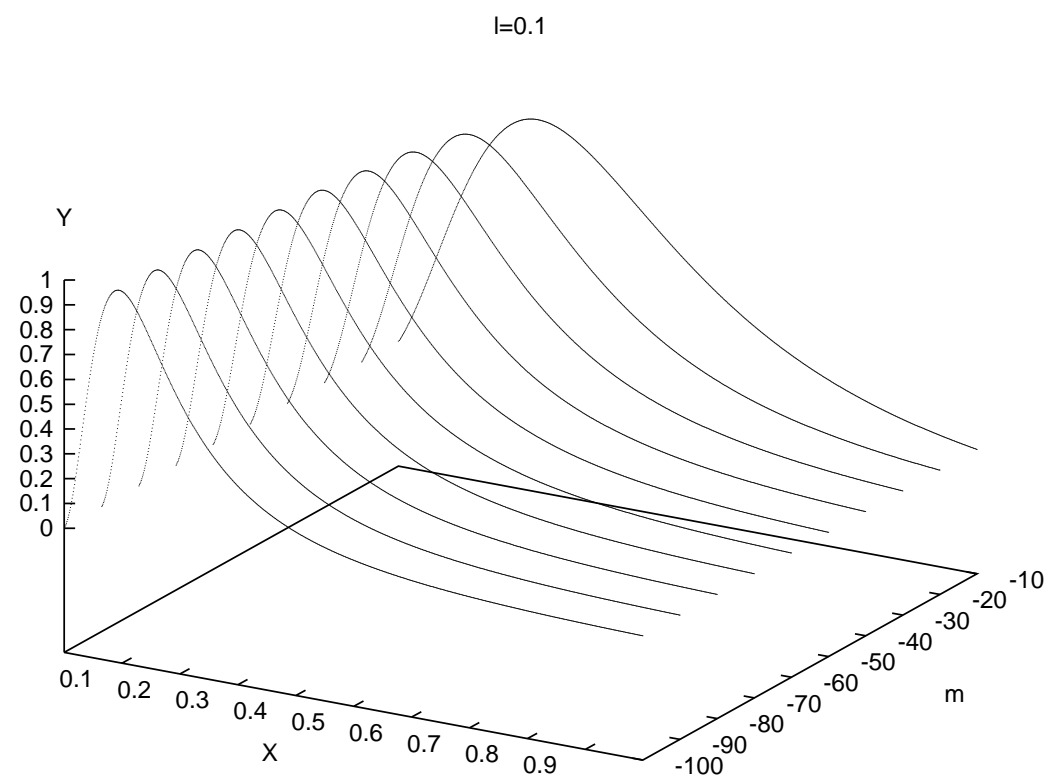


$\mathrm{I}=0.1$

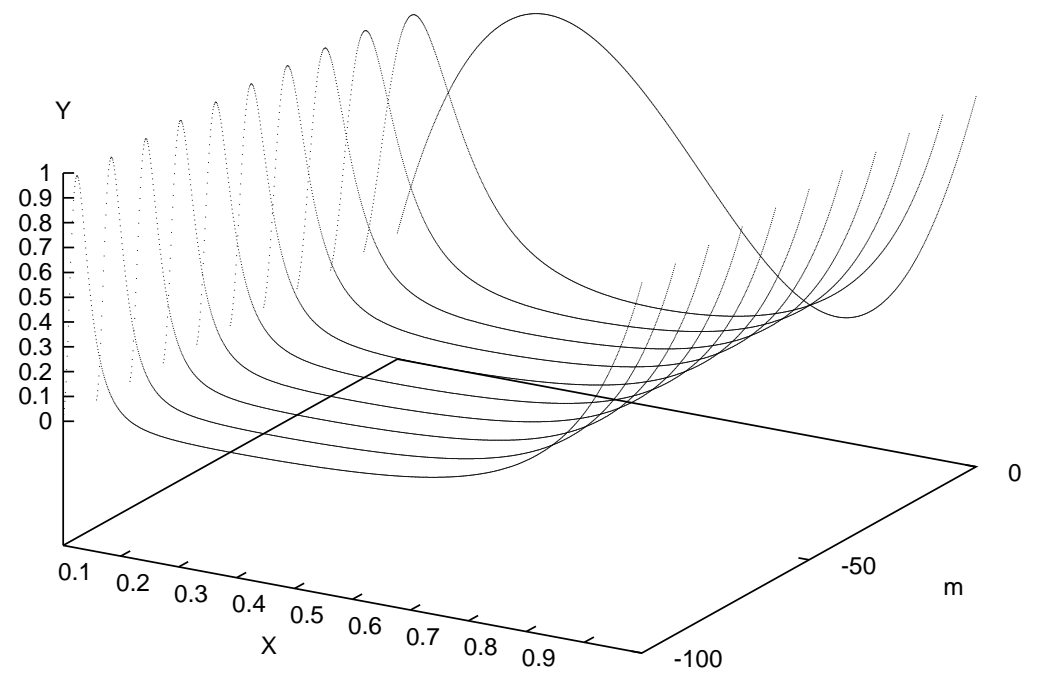

